Relations industrielles

Industrial Relations

\title{
Ouimet, Hélène et Pierre Laporte, Travail plus. Le travail et
} vos droits

\section{Diane Veilleux}

Volume 49, numéro 2, 1994

URI : https://id.erudit.org/iderudit/050948ar

DOI : https://doi.org/10.7202/050948ar

Aller au sommaire du numéro

\section{Éditeur(s)}

Département des relations industrielles de l'Université Laval

\section{ISSN}

0034-379X (imprimé)

1703-8138 (numérique)

Découvrir la revue

Citer ce compte rendu

Veilleux, D. (1994). Compte rendu de [Ouimet, Hélène et Pierre Laporte, Travail plus. Le travail et vos droits]. Relations industrielles / Industrial Relations, 49(2), 415-416. https://doi.org/10.7202/050948ar

Tous droits réservés (C Département des relations industrielles de l'Université Laval, 1994
Ce document est protégé par la loi sur le droit d'auteur. L'utilisation des services d'Érudit (y compris la reproduction) est assujettie à sa politique d'utilisation que vous pouvez consulter en ligne.

https://apropos.erudit.org/fr/usagers/politique-dutilisation/ 
Travail Plus - Le travail et vos droits, par Hélène Oumet et Pierre LAPORTE, Montréal, Wilson \& Lafleur Ltée, 1993, 398 p., ISBN 2-89127-274-9.

Le volume Travail Plus est un ouvrage à caractère didactique qui accompagne le cours télévisé « Le travail et vos droits ». Dans ce volume, les auteurs ont voulu présenter, en treize chapitres, le cadre juridique général des relations du travail au Québec et rendre cette matière accessible aux personnes intéressées au droit du travail. Il ne fait aucun doute que les auteurs ont atteint leurs objectifs parce qu'ils ont réussi à exposer de façon simple mais précise les principales règles de droit qui régissent les rapports individuels et collectifs du travail. Le volume est accessible autant par la forme schématique et le language clair du propos que par le contenu qui expose les règles de droit les plus importantes en droit du travail. En effet, les auteurs n'ont pas alourdi l'exposé en s'abstenant de présenter les approches théoriques, les critiques ou les difficultés d'interprétation et d'application de certaines règles de droit. Toutefois, ils ont pris soin de placer à la fin de chaque chapitre une bibliographie des principaux volumes et des articles des revues spécialisées pertinents au sujet traité. Par sa forme et son contenu, le volume Travail Plus s'adresse d'abord aux non initiés au droit du travail. Cependant, il peut être tout aussi utile comme outil de référence de base aux professionnels dans le domaine des relations du travail.

L'ouvrage séduit par sa forme parce que d'une part, il est très bien structuré. En effet, une table des matières apparaît au début de chaque chapitre et présente en séquence le contenu de la matière étudiée. D'autre part, l'ouvrage capte facilement l'attention visuelle du lecteur ou de la lectrice. Ainsi, l'indication des références à la loi, au règlement ou à la jurisprudence pertinentes est placée en marge du texte. Ce procédé permet donc facilement de référer à la source formelle de la règle de droit exposée. Les auteurs utilisent aussi fréquemment (presque à chaque page) la marge pour mettre en évidence un principe ou une règle de droit importante. Par ailleurs, l'ouvrage didactique comprend beaucoup d'exemples de cas ou de procédures qui illustrent la matière étudiée. Les auteurs ont également placé à la fin de chaque chapitre une série d'exercices et de réponses permettant à l'usager de procéder à son auto-évaluation.

Le volume présente de façon sommaire et cohérente le contenu à jour des principales lois qui s'appliquent dans le domaine des relations du travail et de la jurisprudence qui en précise la portée. Ainsi les deux premiers chapitres du volume situent le champ d'application des lois du travail du Québec, en précisant la définition de salarié et la compétence de la province de Québec, tout comme celle des autres provinces canadiennes, de légiférer en matière de relations du travail. Le troisième chapitre traite du contrat individuel de travail. Les auteurs y exposent particulièrement les conditions de sa formation, sa durée, les obligations respectives de l'employeur et de l'employé, la résiliation du contrat et les recours susceptibles d'être exercés à la suite de l'inexécution ou de la résiliation du contrat de travail. Le quatrième chapitre décrit les normes minimales du travail et les différents recours relatifs à la protection de l'emploi prévus dans la Loi sur les normes du travail.

Le Code du travail qui constitue l'encadrement juridique général des rapports collectifs du travail est ensuite présenté du cinquième au dixième chapitre. Les auteurs traitent alors de la liberté d'association et de la protection de l'exercice de cette liberté. Ils expliquent la procédure d'accréditation qui permet au synaicat majoritaire d'être 
légalement reconnu comme représentant exclusif des salariés de l'unité de négociation. Une fois accrédité, le syndicat devra négocier une convention collective avec l'employeur. Ainsi, les auteurs font part des règles qui régissent le déroulement de la négociation collective et des différents modes de règlement des conflits qui peuvent être mis en œuvre au moment de cette négociation. Ils terminent l'étude du Code du travail par un exposé des règles applicables à la convention collective et au règlement des griefs visant son interprétation et son application.

Le onzième chapitre porte sur trois régimes spéciaux du travail. Les auteurs abordent d'abord l'étude du régime général d'extension juridique de la convention collective institué par la Loi sur les décrets de convention collective. Cette loi permet au gouvernement d'étendre par décret, à un secteur d'activités donné et dans une région déterminée, certaines conditions de travail prévies dans une convention collective lorsque la demande lui en est faite par l'une des parties à cette convention. Les auteurs étudient ensuite le régime de relations du travail applicable aux salariés de l'industrie de la construction. Les salariés de ce secteur d'activités sont exclus de l'application du Code $d u$ travail et sont soumis à un régime particulier de négociation collective sectorielle. Les auteurs terminent le onzième chapitre par l'étude du régime des relations du travail applicable aux secteurs public et parapublic. La négociation collective dans ces secteurs d'activités est centralisée à l'échelle provinciale et est régie par des dispositions particulières prévues dans le Code du travail et dans différentes lois spécifiques.

Le douzième chapitre vise les pièces législatives majeures en santé et sécurité du travail. Ainsi sont sommairement étudiées La Loi sur la santé et la sécurité du travail qui a pour objet la prévention des accidents du travail et la Loi sur les accidents du travail et les maladies professionnelles qui institue un régime provincial d'indemnisation des accidents du travail. Le dernier chapitre complète la présentation générale du cadre juridique des relations du travail par l'examen des chartes canadienne et québécoise qui affirment les libertés et les droits fondamentaux de la personne et interdisent l'exercice de mesures discriminatoires.

En somme, le volume Travail Plus répond parfaitement aux buts poursuivis par ses auteurs. Cet ouvrage bien conçu se lit facilement et favorise l'acquisition de connaissances juridiques générales en matière de relations du travail au Québec.

Diane VeilleuX

Université de Montréal

Work and Health, edited by M. QuINLAN, Melbourne, Macmillan Education Australia, 1993, 353 p., ISBN 0-7329-1486-8.

The study of occupational health and safety lends itself to a multi-disciplinary approach. To explore the causes of injury and disease and to design and assess regulatory responses may require the insights of economics, sociology, psychology, political science and law. Most books on occupational health and safety, including edited collections, tend to approach the subject from a single disciplinary perspective; better ones may include two. This fine collection breaks with that tradition. It assembles articles 